\title{
Inpatient Outcomes of Surgical Treatment for Spinal Tuberculosis: 10-Year Results from the US National Inpatient Sample
}

\author{
Guangwei SUN ${ }^{1 *}$, Jingjing $\mathrm{WU}^{2 *}$, Caibin $\mathrm{GAO}^{3}$, Qiang $\mathrm{LIU}^{4}$, Hucheng YUAN \\ 'Linfen People's Hospital, Department of Orthopaedics, Shanxi, China \\ 'Linfen People's Hospital, Department of Blood transfusion, Shanxi, China \\ ${ }^{3}$ General Hospital of Ningxia Medical University, Department of Neurosurgery, Ningxia Hui Autonomous Region, China \\ ${ }^{4}$ YongKang Hospital of Traditional Chinese Medicine, Department of Orthopaedics, Zhejiang, China \\ ${ }^{5}$ General Hospital of Ningxia Medical University, Department of Spinal Surgery, Ningxia Hui Autonomous Region, China \\ ${ }^{*}$ Guangwei Sun and Jingjing Wu are co-first authors.
}

Corresponding author: Guangwei SUN guangweisu34@edtsbm.cn

\section{ABSTRACT}

AIM: To evaluate the association between in-hospital outcomes of patients with spinal tuberculosis and different surgical approaches.

MATERIAL and METHODS: This population-based, retrospective observational study analyzed data of hospitalized patients undergoing surgical treatment for spinal tuberculosis in the United States who were identified in the US Nationwide Inpatient Sample (NIS) between 2005 and 2014. The study cohort was stratified by posterior-only, anterior-only, and combined surgical approaches. Logistic and linear regression analyses were performed to evaluate associations between surgical approaches and patient outcomes.

RESULTS: Significant differences were found in postoperative complications, number of instrumented levels, and comorbidity scores (all $p \leq 0.033$ ) between patients who received different surgical approaches. A univariate analysis demonstrated the combined approach was associated with significantly increased odds of postoperative complications compared with the posterior-only approach. This association remained significant when following multivariate analysis after adjustments. For patients who received surgery at the lumbosacral level, the anterior-only approach and combined approach were both associated with significantly increased odds of postoperative complications compared with the posterior-only approach. Among patients who received surgery at the lumbosacral level, multivariate analysis showed that anterior-only and combined approaches were associated with significantly longer length of stay.

CONCLUSION: Among patients with spinal tuberculosis who are undergoing surgical treatment, a posterior-only approach was associated with fewer complications and shorter length of stay compared with an anterior-only or combination approaches when performed at the lumbo-sacral spinal level.

KEYWORDS: Spinal tuberculosis, Pott's disease, Spinal fusion, Anterior approach, Posterior approach, National Inpatient Sample (NIS)

Guangwei SUN 무: :0000-0001-6796-8372

Jingjing WU (10): 0000-0003-3747-7665

Caibin GAO (10): 0000-0002-0059-4123
Qiang LiU (1) : 0000-0002-2172-7028

Hucheng YUAN (1) : 0000-0003-4189-9684 


\section{INTRODUCTION}

pinal tuberculosis is the most frequent type of extrapulmonary tuberculosis (TB), occurring in approximately $1 \%-3 \%$ of all patients with TB (1), and accounting for half of all cases of musculoskeletal or osteoarticular TB $(5,9)$. Since spinal TB is destructive and often leads to spinal malformation and paralysis (5), it is considered a threat to the musculoskeletal system (13). TB and extrapulmonary TB have been as early as 1000-600 BC, and with the emergence of HIV/AIDS and increased migration worldwide, the disease has surfaced in countries where it was once eradicated (9). The World Health Organization (WHO) describes TB as a major global health problem that affects approximately 10 million individuals each year. TB is a leading cause of death from a single infective agent and results in more deaths annually than HIV/AIDS (20). Although some authors have reported an increased incidence of TB since 1995 (5), others have reported the incidence between 2002 and 2011 has decreased to one case per two-million individuals in 2011 , a relatively minor decrease (2).

Management of patients with spinal TB depends on the stages of neurological involvement and progression through aggressively increasing motor deficits; accordingly, management measures include a mix of rest, braces, traction, chemotherapy, and surgery (9). Antituberculosis therapy is still considered the cornerstone treatment for spinal TB and chemotherapeutic agents may be administered for up to six months $(5,19,22)$. Nevertheless, even though early identification of the causative pathogen and administration of chemotherapy agents have helped to prevent severe deformities in patients with spinal TB, surgical treatment remains an important component of treatment, and is often required for decompression, correction of kyphosis, and maintaining spinal stability $(10,19,22)$. Spinal TB is a major cause of kyphotic deformity because it affects the anterior elements of the vertebral column in $90 \%$ of affected individuals. Even after chemotherapy has inactivated the infection, vertebral collapse may occur, leading to some degree of deformity, especially in children (16). Common indications for surgery include the patients' degree of deformity, neurological deficit, and instability (4). Surgery may also be considered when the diagnosis is in doubt or when kyphosis prevention is needed (9). Various approaches to spinal fusion have been proposed and reported, however the selection of a surgical approach for spinal TB remains controversial because the surgery is expected to facilitate complete debridement, relieve compression on the spinal cord, improve neurologic function, correct kyphosis, and restore spinal stability (21). An anterior surgical approach provides direct access to the lesion and a convenient way to debride and reconstruct the defect (7), although biomechanical stability of the spine may be compromised and residual kyphosis may be present after treatment (21). The combined anterior-posterior surgical approach is noted for overcoming stability issues common to the anterior approach and is being performed more often. Anterior debridement with posterior instrumentation is reported to debride the lesion completely, ensure bone graft fusion, correct kyphosis, reduce recurrence, and prevent future kyphosis (8). However, the combined surgical approach may also lead to longer operative times, increased blood loss, and an increased number of postoperative complications (14). The posterior-only surgical approach has been shown to be safe and effective for patients with spinal TB as long as indications for the procedure are correct. The posterior surgical approach has been suggested to achieve multiple effects of anterior surgery, but with fewer complications (21). Despite the differences noted between surgical approaches, a recent meta-analysis that evaluated anterior vs. posterior surgical approaches for patients with spinal TB reported no significant differences between the two approaches in regard to operative times, blood loss, length of stay, total fusion time, and loss of Cobb angle during follow-up (12).

Comparative studies examining the relationship between types of surgery and outcomes, as well as regional differences and surgeon preferences are lacking (11). Although several studies have compared different surgical approaches for the treatment of spinal TB, no standard procedure or an optimum approach has achieved consensus among surgeons $(1,10,12,21)$. In the absence of guidelines for treating spinal TB, it is imperative for clinicians to understand which treatment strategy and surgical approach will prevent neurological disability and minimize spinal deformity. Therefore, to help clarify the benefits and drawbacks of the three most commonly used surgical approaches for spinal TB, we currently aimed to evaluate the characteristics and in-hospital outcomes of patients with spinal TB who underwent spinal fusion using different surgical approaches, and to analyze the associations between patient outcomes and these surgical approaches.

\section{MATERIAL AND METHODS}

\section{Study Design and Ethical Considerations}

This is a population-based, retrospective observational study using data extracted from the Nationwide Inpatient Sample (NIS), which is the largest all-payer U.S. inpatient care database and represents $20 \%$ of inpatient admissions from 45 states that participate in the program. The NIS database was developed by the US Healthcare Cost and Utilization Project (HCUP) and is maintained by the Agency for Healthcare Research and Quality (AHRQ). It is a continuous survey that draws data from 1,050 participating hospitals, including over one-hundred clinical and nonclinical items (ie, primary and secondary diagnoses and procedures, admission/discharge status, patient demographics, length of stay, and hospital characteristics) from approximately 8 million hospital stays every year (15). The NIS also provides statistical weights that allow estimates of national case volumes to be generalized.

All data was obtained through request to the HCUP Central Distributor (https://www.distributor.hcup-us.ahrq.gov/). We conformed to the data-use agreement for the NIS and received certificate number HCUP-873EVW39K. The study protocol was reviewed and approved by the internal review board of our institution. Since previously collected de-identified data was used, additional informed consent was waived for the present study. 


\section{Study Population}

Patients hospitalized in the United States with a diagnosis of TB were identified in the NIS database between 2005 and 2014 using Clinical Classifications Software (CCS) code \#1(6), a tool provided by HCUP for clustering patient diagnoses and procedures into a manageable number of clinically meaningful categories. Patients with spinal TB were then identified via International Classification of Diseases, Ninth Revision (ICD9) diagnosis code of 015.00, 015.01, 015.02, 015.03, 015.04, 015.05 , and 015.06 . Patients who underwent spinal fusion with a known approach were then identified via ICD-9 procedural codes $81.00-81.08$ as the primary cohort. The study cohort was further stratified by 3 different surgical approaches: posterior only (ICD-9 procedural code 81.03 , 81.05, 81.07, 81.08 ), anterior only (ICD-9 procedural code 81.02, 81.04, 81.06), and combined approaches.

\section{Primary Measures and End Points}

Patients' demographic characteristics included age, gender, race, income by ZIP code, and insurance status (primary payer). Clinical characteristics included admission type (elective vs. emergent), surgical site, number of instrumented levels, and primary or revision surgery performed.

Comorbidities were identified according to $A H R Q$ comorbidity measures in the database determined with ICD-9 diagnostic codes using algorithms validated with the Elixhauser comorbidity scores (3). Hospital-related characteristics (bed size/ location/teaching status/hospital region and annual caseload of spinal fusion) were included in patient data extracted from the NIS database.

The primary endpoints were postoperative complications and length of hospital stay.

Postoperative complications were defined by the following ICD-9 codes and Clinical Classifications Software (CCS) codes: Cardiovascular complications: 997.1, 997.02, 997.09, 998.0, 100CCS; Bleeding complications: 285.1, 998.1998.2; Respiratory complications and pneumonia: 518.5, 518.81, 997.3, 122CCS; Acute renal failure: 584, 157CCS, V45.1; Infection/sepsis: 998.5, 995.9; Deep vein thrombosis (DVT) / Pulmonary embolism: 451.11, 451.19, 451.2, 451.81-84, 451.89, 451.9, 453.40-42, 453.8, 453.9, 997.2; Wound complications: 998.12-998.13, 998.3, 998.5; Device complications: 996.1, 996.62, 996.74, 998.2, 998.4, 998.7; Other complications: 997.0, 997.4, 997.5, 997.9, 998.6, 998.8, 998.9; and in-hospital death.

\section{Statistical Analysis}

Continuous variables are presented as means with standard error (SE) were tested using an analysis of variance (ANOVA). Categorical variables are presented as weighted percentages and tested by Chi-square test. Logistic regression analyses and linear regression analyses were conducted to evaluate associations between approaches and outcomes (postoperative complications and length of stay) in the whole population, thoraco-lumbar and lumbosacral populations. Variables that were significantly associated with outcomes in univariate regression models (such as postoperative complications and length of stay), were added into the multivariate regression models. The mean, SE, proportions, all tests and regression models were applied with discharge weights to satisfy the HCUP-NIS sampling method. Two-sided $\mathrm{p}<0.05$ was considered statistically significant. Statistical analyses were performed using the statistical software package SAS version 9.4 (SAS Institute Inc., Cary, NC, USA).

\section{RESULTS}

\section{Study Population}

A total of 121,284 patients from the 2005-2014 HCUP-NIS database (weighted $n=599,706$ ) were diagnosed with TB, of whom 1,346 (weighted $n=6,688$ ) were diagnosed with spinal TB. Among these patients, 161 patients (weighted $n=796$ ) who received surgery for spinal TB were enrolled in the study and their data were analyzed and compared based on surgical approach.

\section{Descriptive Statistics}

Patients' demographic and clinical characteristics and surgical outcomes are summarized in Tables I and II, along with hospital characteristics. Significant differences were found in postoperative complications, number of instrumented levels, Elixhauser comorbidity scores, hospital caseload of spinal fusion, income by hospital location (ZIP code), and patients' insurance status (all $\mathrm{p} \leq 0.033$ ) between patients receiving posterior-only, anterior-only, or combined approaches to surgery. Patients who received the combined surgical approach had an increased number of spinal levels instrumented compared with the other groups $(61.5 \%$ vs. $58.8 \%$ vs. $22.8 \%$, combined vs. posterior vs. anterior, respectively, $p<0.001)$. An increased number of patients who received the anterior surgical approach had Elixhauser comorbidity scores equal to 1 compared with the other groups $(41.7 \%$ vs. $22.6 \%$ vs. $23.6 \%$, anterior vs. posterior vs. combined, respectively, $p=0.007$ ) Patients who received the combined surgical approach had a greater percentage of postoperative complications compared with the other groups $(63.1 \%$ vs. $41.0 \%$ vs. $34.8 \%$, combined vs. posterior vs. anterior, respectively, $\mathrm{p}=0.002$ ) (Table II).

In hospitals that reported the highest quartile of annual spinal fusion procedures ( $\geq 457$ cases), a greater percentage of patients underwent an anterior-only surgical approach compared with the other groups ( $41.4 \%$ vs. $18.8 \%$ vs. $26.4 \%$, anterior vs. posterior vs. combined, respectively, $\mathrm{p}<0.001$ ) More patients who underwent surgery via a posterior-only approach had the least household income $\left(0-25^{\text {th }}\right.$ percentile) compared with the other groups $(43.8 \%$ vs. $35.1 \%$ vs. $28.4 \%$, posterior vs. anterior vs. combined, respectively, $\mathrm{p}=0.003$ ) More patients who underwent surgery via a posterior approach had Medicare or Medicaid insurance compared with the other groups $(71.2 \%$ vs. $53.3 \%$ vs. $56.3 \%$, posterior vs. anterior vs. combined, respectively, $\mathrm{p}=0.033$ ) (Tables I and II).

\section{Associations Between Postoperative Complications and Surgical Approach}

Univariate analysis showed that the combined approach was associated with significantly increased odds for postoperative 
Table I: Patient Demographic and Clinical Characteristics Stratified by Surgical Approach

\begin{tabular}{|c|c|c|c|c|c|}
\hline & Overall & Posterior only & Anterior only & Combined & $p$ \\
\hline & wt $\%$ & wt $\%$ & wt \% & wt $\%$ & \\
\hline \multicolumn{6}{|l|}{ Patient characteristics } \\
\hline Age & & & & & 0.061 \\
\hline$<65$ years & 72.3 & 66.3 & 77.1 & 78.6 & \\
\hline$\geq 65$ years & 27.7 & 33.7 & 22.9 & 21.4 & \\
\hline Gender, female & 40.2 & 41.2 & 47.7 & 34.6 & 0.325 \\
\hline Race & & & & & 0.197 \\
\hline White & 29.1 & 27.9 & 21.1 & 35.1 & \\
\hline Black & 26.4 & 24.4 & 26.1 & 29.7 & \\
\hline Other & 44.5 & 47.7 & 52.8 & 35.2 & \\
\hline Income by ZIP code & & & & & 0.003 \\
\hline $0-25^{\text {th }}$ percentile & 37.0 & 43.8 & 35.1 & 28.4 & \\
\hline $26^{\text {th }}$ to $50^{\text {th }}$ percentile & 22.9 & 19.6 & 26.2 & 25.9 & \\
\hline $51^{\text {st }}$ to $75^{\text {th }}$ percentile & 16.4 & 14.0 & 3.6 & 26.9 & \\
\hline $76^{\text {th }}$ to $100^{\text {th }}$ percentile & 23.6 & 22.5 & 35.1 & 18.8 & \\
\hline Insurance status & & & & & 0.033 \\
\hline Medicare/Medicaid & 63.1 & 71.2 & 53.3 & 56.3 & \\
\hline Private including $\mathrm{HMO}$ & 21.4 & 13.7 & 29.2 & 28.6 & \\
\hline Self-pay/no charge/other & 15.5 & 15.1 & 17.5 & 15.1 & \\
\hline Surgical site & & & & & NA \\
\hline Cervical & 7.5 & 5.0 & 21.2 & 3.8 & \\
\hline Thoraco-lumbar & 59.1 & 69.3 & 54.4 & 46.3 & \\
\hline Lumbosacral & 25.7 & 23.2 & 24.4 & 30.3 & \\
\hline Mixed & 7.6 & 2.5 & 0.0 & 19.6 & \\
\hline Number of instrumented levels & & & & & $<0.001$ \\
\hline$<4$ & 36.0 & 31.8 & 73.0 & 22.0 & \\
\hline $4-8$ & 53.2 & 58.8 & 22.8 & 61.5 & \\
\hline $9+$ & 10.7 & 9.4 & 4.2 & 16.5 & \\
\hline Admission type & & & & & 0.073 \\
\hline Elective & 36.5 & 30.9 & 49.2 & 38.0 & \\
\hline Emergent & 63.5 & 69.1 & 50.8 & 62.0 & \\
\hline Primary or revision surgery & & & & & NA \\
\hline Primary & 95.1 & 93.8 & 100.0 & 94.3 & \\
\hline Revision & 4.9 & 6.2 & 0.0 & 5.7 & \\
\hline Elixhauser comorbidity score & & & & & 0.007 \\
\hline 0 & 16.3 & 20.2 & 11.1 & 13.3 & \\
\hline 1 & 26.4 & 22.6 & 41.7 & 23.6 & \\
\hline 2 & 17.2 & 14.9 & 24.1 & 16.7 & \\
\hline 3 & 14.8 & 16.1 & 2.5 & 19.6 & \\
\hline $4+$ & 25.4 & 26.3 & 20.6 & 26.7 & \\
\hline
\end{tabular}


Table I: Cont.

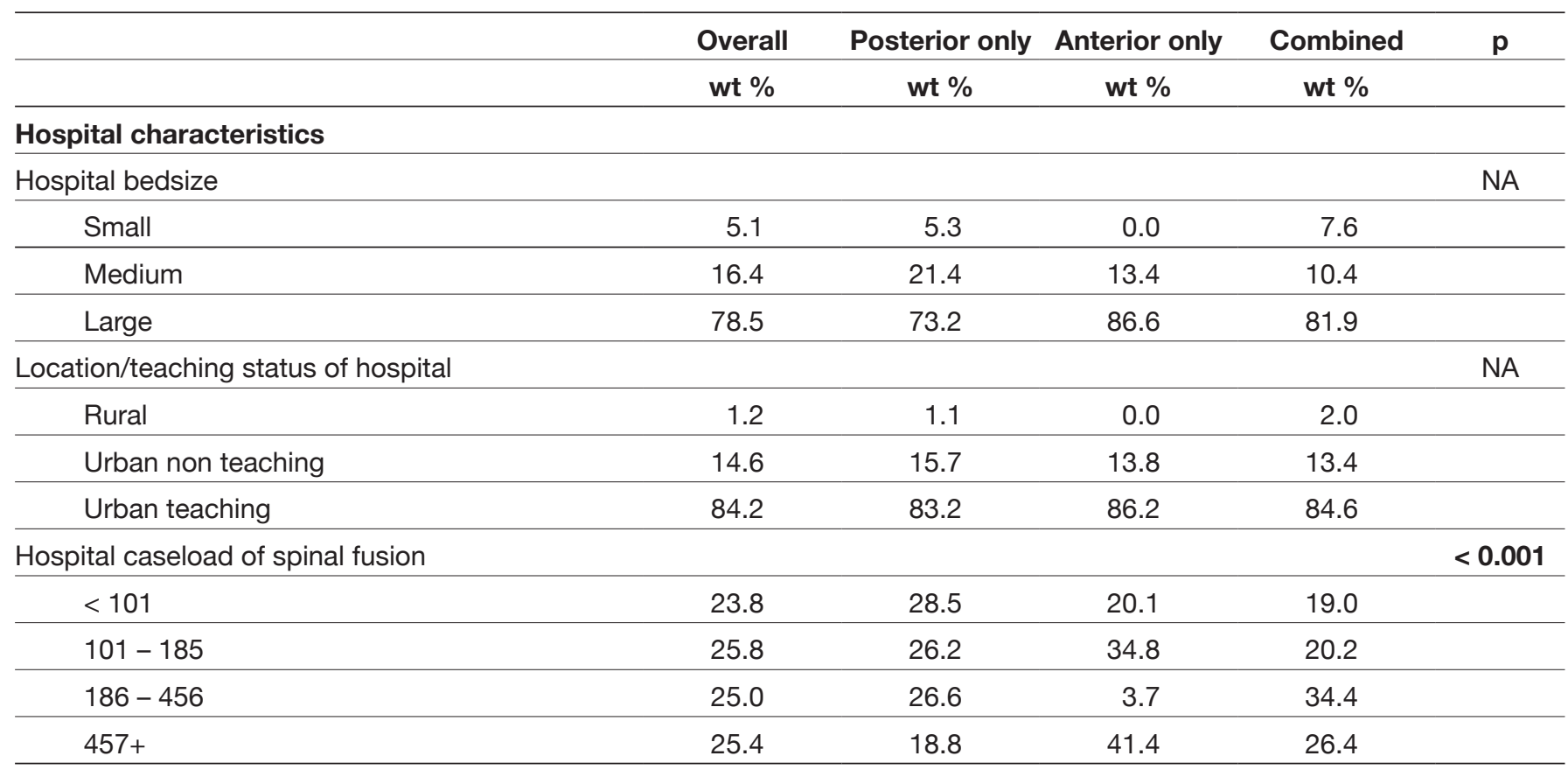

wt: Weighted. Boldface indicates significant difference, $p<0.05$.

Table II: Patients' Clinical Outcomes Stratified by Surgical Approach

\begin{tabular}{|c|c|c|c|c|c|}
\hline & Overall & Posterior only & Anterior only & Combined & $\mathbf{p}$ \\
\hline \multicolumn{6}{|l|}{ Outcomes } \\
\hline Postoperative complication & 47.1 & 41.0 & 34.8 & 63.1 & 0.002 \\
\hline Cardiovascular complications & 2.4 & 3.5 & 0.0 & 2.1 & \\
\hline Infection/ sepsis & 8.7 & 8.8 & 3.1 & 11.7 & \\
\hline DVT/ pulmonary embolism & 6.1 & 8.6 & 0.0 & 5.7 & \\
\hline Bleeding complications / transfusion & 17.0 & 8.7 & 13.9 & 31.2 & \\
\hline Wound complications & 3.1 & 3.7 & 0.0 & 3.8 & \\
\hline In-hospital mortality & 2.4 & 4.9 & 0.0 & 0.0 & \\
\hline Length of stay, days & $16.98 \pm 0.84$ & $16.96 \pm 0.79$ & $13.97 \pm 1.86$ & $18.69 \pm 1.94$ & 0.367 \\
\hline
\end{tabular}

wt: Weighted. Boldface indicates significant difference, $p<0.05$.

complications compared with the posterior-only surgical approach among the overall population $(\mathrm{OR}=2.11,95 \% \mathrm{Cl}$ $=1.12-3.98, p=0.021$ ) (Table III). The association remained significant after adjusting for gender, number of instrumented levels, Elixhauser comorbidity scores, hospital bed size, and location/teaching status of the hospital $(\mathrm{aOR}=2.28,95 \% \mathrm{Cl}=$ $1.11-4.71, p=0.025)$. Since the majority of patients underwent surgeryat the thoraco-lumber or lumbosacral level $\mathbf{5 9 . 1 \%}$ and $25.7 \%$, respectively, Table I), associations between postoperative complications and surgical approaches at these specific sites were evaluated further. For patients who receivedsurgery at the lumbosacral level, both the anterioronly approach $(\mathrm{OR}=9.33,95 \% \mathrm{Cl}=1.93-45.04, \mathrm{p}=0.005)$ and the combined approach $(\mathrm{OR}=11.27,95 \% \mathrm{Cl}=4.54-27.95$, 
Table III: Association Between Surgical Approaches and Postoperative Complications Stratified by Surgical Site (Univariate and Multivariate Analyses)

\begin{tabular}{|c|c|c|c|c|c|c|}
\hline & \multicolumn{6}{|c|}{ Univariate analysis } \\
\hline & \multicolumn{2}{|l|}{ Overall } & \multicolumn{2}{|c|}{ Thoraco-lumbar } & \multicolumn{2}{|c|}{ Lumbosacral } \\
\hline & OR (95\% Cl) & $\mathbf{p}$ & OR $(95 \% \mathrm{Cl})$ & $\mathbf{p}$ & OR $(95 \% \mathrm{Cl})$ & $\mathbf{p}$ \\
\hline \multicolumn{7}{|l|}{ Approach } \\
\hline Posterior only & 1 & & 1 & & 1 & \\
\hline Anterior only & $0.73(0.34,1.60)$ & 0.433 & $0.56(0.26,1.19)$ & 0.133 & $9.33(1.93,45.04)$ & 0.005 \\
\hline \multirow[t]{3}{*}{ Combined } & $2.11(1.12,3.98)$ & 0.021 & $1.44(0.60,3.46)$ & 0.410 & $11.27(4.54,27.95)$ & $<0.001$ \\
\hline & \multicolumn{6}{|c|}{ Multivariate analysis } \\
\hline & aOR $(95 \% \mathrm{Cl})^{\mathrm{a}}$ & $\mathbf{p}$ & aOR $(95 \% \mathrm{Cl})^{b}$ & p & $\operatorname{aOR}(95 \% \mathrm{Cl})^{\mathrm{c}}$ & p \\
\hline
\end{tabular}

Approach

\begin{tabular}{lcccccc}
\hline Posterior only & 1 & \multicolumn{1}{c}{1} \\
\hline Anterior only & $1.13(0.40,3.20)$ & 0.816 & $1.48(0.42,5.26)$ & 0.542 & $25.04(7.06,88.76)$ & $<0.001$ \\
\hline Combined & $2.28(1.11,4.71)$ & $\mathbf{0 . 0 2 5}$ & $0.78(0.23,2.68)$ & 0.690 & $15.25(5.97,38.99)$ & $<0.001$ \\
\hline
\end{tabular}

a Model was adjusted by gender, number of instrumented levels, Elixhauser comorbidity score, hospital bed size, and location/teaching status of hospital.

${ }^{b}$ Model was adjusted by insurance status, number of instrumented levels, admission type, Elixhauser comorbidity score, and hospital caseload of spinal fusion.

' Model was adjusted by gender, race, and location/teaching status of hospital.

OR: odds ratio, Cl: confidence interval.

Boldface indicates significant association, $p<0.05$.

$\mathrm{p}<0.001)$ were significantly associated with increased odds of postoperative complications compared with the posterioronly surgical approach group (Table III). Both the anterior-only surgical approach group $(\mathrm{aOR}=25.04,95 \% \mathrm{Cl}=7.06-88.76$, $\mathrm{p}<0.001)$ and the combined surgical approach group (aOR = $15.25,95 \% \mathrm{Cl}=5.97-38.99, \mathrm{p}<0.001$ ) remained significantly associated with postoperative complications among patients who received surgery at the lumbosacral level after adjusting for gender, race, and location/teaching status of the hospital. However, no significant associations were identified between the surgical approaches and postoperative complications among patients who received surgery at the thoraco-lumbar level in either univariate or multivariate models (Table III).

\section{Associations Between Surgical Approaches and Length of Stay}

Univariate analysis showed no significant associations between surgical approaches and length of stay (Table IV). Among the overall population, after adjusting for race, number of instrumented levels, admission type, Elixhauser comorbidity scores, hospital bed size, insurance status, and location/teaching status of the hospital, the anterior-only surgical approach was associated with significantly shorter length of stay compared with the posterior-only surgical approach group (beta $=-1.78,95 \% \mathrm{Cl}=-2.57,-1.00, \mathrm{p}<$ $0.001)$, while the combined surgical approach was associated with significantly longer length of stay (beta $=2.69,95 \% \mathrm{Cl}=$ $1.84,3.53, p<0.001)$. Among patients who received surgery at the thoraco-lumbar level, multivariate analysis revealed that the anterior-only surgical approach was associated with significantly shorter length of stay compared with the posterior-only surgical approach group (beta $=-1.63,95 \% \mathrm{Cl}$ $=-2.27,-0.99, \mathrm{p}<0.001)$. Among patients who received surgery at the lumbosacral level, multivariate analysis demonstrated that anterior-only and combined surgical approaches were associated with significantly longer length of stay (beta $=4.57$, $95 \% \mathrm{Cl}=3.28,5.86, \mathrm{p}<0.001$; beta $=0.64,95 \% \mathrm{Cl}=0.60$, $0.68, p<0.001$, respectively) (Table IV).

\section{DISCUSSION}

In the present study, the outcome data for patients with spinal TB who underwent surgical treatment were analyzed on the basis of surgical approach-anterior only, posterior only, or combined approach. For the overall population, results revealed that the combined surgical approach was not only associated with a longer length of stay compared with the posterior-only surgical approach, but it was also associated with more postoperative complications. Differences were observed when data was stratified by surgery site. For patients who received surgery at the lumbo-sacral level, the anterior-only and the combined surgical approaches had greater postoperative complications and longer length of stay compared with the posterior-only approach. For patients who received surgery at the thoraco-lumbar level, no significant differences were found in postoperative complications 
Table IV: Association Between Different Surgical Approaches and Length of Stay Stratified by Surgical Site (Univariate and Multivariate Analyses)

\begin{tabular}{|c|c|c|c|c|c|c|}
\hline & \multicolumn{6}{|c|}{ Univariate analysis } \\
\hline & \multicolumn{2}{|l|}{ Overall } & \multicolumn{2}{|c|}{ Thoraco-lumbar } & \multicolumn{2}{|c|}{ Lumbosacral } \\
\hline & Beta $(95 \% \mathrm{Cl})$ & $\mathbf{p}$ & Beta $(95 \% \mathrm{Cl})$ & p & Beta $(95 \% \mathrm{Cl})$ & $\mathbf{p}$ \\
\hline \multicolumn{7}{|l|}{ Approach } \\
\hline Posterior only & 1 & & 1 & & 1 & \\
\hline Anterior only & $-2.99(-8.05,2.07)$ & 0.244 & $-3.64(-9.72,2.45)$ & 0.237 & $3.25(-10.50,17.00)$ & 0.633 \\
\hline \multirow[t]{4}{*}{ Combined } & $1.73(-3.42,6.89)$ & 0.507 & $-1.84(-6.92,3.24)$ & 0.473 & $2.11(-4.70,8.93)$ & 0.531 \\
\hline & \multicolumn{6}{|c|}{ Multivariate analysis } \\
\hline & \multicolumn{2}{|l|}{ Overall } & \multicolumn{2}{|c|}{ Thoraco-lumbar } & \multicolumn{2}{|c|}{ Lumbosacral } \\
\hline & Beta $(95 \% \mathrm{Cl})^{\mathrm{a}}$ & p & Beta $(95 \% \mathrm{Cl})^{\mathrm{b}}$ & p & Beta $(95 \% \mathrm{Cl})^{\mathrm{c}}$ & $\mathrm{p}$ \\
\hline
\end{tabular}

Approach

\begin{tabular}{lcccccc}
\hline Posterior only & 1 & 1 & 1 & \\
\hline Anterior only & $-1.78(-2.57,-1.00)$ & $<0.001$ & $-1.63(-2.27,-0.99)$ & $<0.001$ & $4.57(3.28,5.86)$ & $<0.001$ \\
\hline Combined & $2.69(1.84,3.53)$ & $<0.001$ & $-1.59(-3.67,0.49)$ & 0.131 & $0.64(0.60,0.68)$ & $<0.001$ \\
\hline
\end{tabular}

a Model was adjusted by race, insurance status, number of instrumented levels, admission type, Elixhauser comorbidity score, hospital bed size, and location/teaching status of hospital.

${ }^{b}$ Model was adjusted by race, insurance status, number of instrumented levels, admission type, Elixhauser comorbidity score, hospital bed size, and location/teaching status of hospital.

c Model was adjusted by race, number of instrumented levels, admission type, and hospital bedsize.

Cl: confidence interval.

Bold face indicates significant association, $p<0.05$.

between the surgical approaches. However, among patients who received surgery at the thoraco-lumbar level, the anterior-only surgical approach was associated with a shorter length of stay compared with those undergoing the posterior-only approach.

In the present study, in the hospitals with a higher caseload of patients with spinal TB, the majority of patients received surgery via the anterior-only approach. The anterior approach was selected primarily for its direct access, optimum visualization, and direct and complete decompression of the cord through anterior radical surgical excision (17). In the present study, differences in postoperative complications between surgical approaches were perhaps more revealing than length of stay. In the overall population, for example, the odds of postoperative complications doubled in the combined approach than in the posterior-only surgical approach group. In addition, both the anterior-only and combined surgical approaches were associated with significantly increased odds of postoperative complications than the posterior-only approach when surgery was at the lumbosacral level. These results are in agreement with previously published reports that suggest a combined surgical approach may lead to longer operative times, increased blood loss, and increased postoperative complications (14). Studies that support the posterior-only surgical approach as safe and effective for patients with spinal
TB, emphasize the indication for the procedure be correct and suggest that the posterior approach readily achieves the multiple effects of anterior surgery and does so with fewer complications (21). Two meta-analyses conducted by Yang et al., one that evaluated safety and efficacy between all three approaches and the other between only anterior and posterior $(17,18)$. Posterior instrumentation appeared to result in similar surgical outcomes as anterior instrumentation in adults with spinal TB-both in correcting the deformity and maintaining that correction without significant complications. Another meta-analysis that included five randomized controlled trials comparing posterior-only with combined anterior-posterior surgical approaches concluded that the posterior approach had the same clinical advantages as the anterior-posterior approach but with less operative time, less blood loss, fewer complications, and shorter length of stay (10). Rajasekaran suggested that surgeons should decide on a preferred approach based on their expertise and preferences, and it was emphasized that refinements to the surgical technique and advanced instrumentation that provides three-column support make prevention and correction of deformity possible using the posterior-only approach (16). Further studies are needed to identify differences in the complication types and rates between anterior and posterior approaches for surgical treatment of spinal TB. 
Our consideration of the sites of surgery revealed differences in results for length of stay and postoperative complications. The majority of patients in the present study received surgery at the thoraco-lumbar or lumbosacral level, which led us to examine any possible associations between postoperative complications, length of stay and surgical approaches among surgeries performed at these sites separately. Among patients who had surgery at the lumbosacral level, multivariate analysis, after adjustments for gender, race, and other covariates, showed that anterior-only and combined surgical approaches were associated with significantly longer length of stay and more postoperative complications compared with the posterior-only approach. No significant associations were identified between surgical approaches and postoperative complications among patients who had surgery at thoracolumbar level. The other two approaches had longer length of stay compared with the posterior-only approach when surgery was at the lumbosacral level. However, at the thoraco-lumbar level, the anterior-only surgical approach had a shorter length of stay. The reasons why these differences exist are unknown. In a review of randomized controlled trials addressing surgical intervention for patients with spinal TB, surgeons were more familiar with the anterior approach for its direct decompression and rigid structural stabilization, but for the thoracic and lumbar spine, posterior-based implants and safe techniques for achieving circumferential decompression and stabilization influenced increased use of posterior approaches. Those authors concluded that the anterior approach was preferred in the cervical and cervicothoracic spine and the posterior approach was preferred in the thoracic and lumbar spine in adults with spinal TB (4).

\section{Limitations}

This study has a few limitations regarding the interpretation of results, including data that were analyzed retrospectively, limiting any inference of causation. In addition, although the comprehensive NIS database is an important strength of this study, patients with a diagnosis of spinal TB were identified using the ICD-9 coding system and coding errors may have occurred. Comorbidities also were identified through the ICD coding system and the severity of comorbidities could not be accounted for in the analysis. Although hospital caseload was included in the analyses, the individual surgeon's experience was unknown and thus could not be accounted for. The reason for choosing anterior or posterior surgical approaches, data on preoperative chemotherapy, operative time, blood loss, degrees of deformity, and maintenance of spinal stability after discharge were not available in the NIS database and could have made important differences in the interpretation of the results. Other possible confounding variables that were not included in the NIS database that were not included in the analyses, which may ultimately have influenced results.

\section{CONCLUSION}

For patients with spinal TB who undergo surgical treatment, a posterior-only approach is associated with fewer complications and shorter length of stay compared with an anterior-only or combined surgical approaches when performed at the lumbo- sacral level. Further well-designed prospective studies are warranted in order to confirm the results of the present study and to optimize the selection of surgical approach in this patient population.

\section{REFERENCES}

1. Cui X, Li LT, Ma YZ: Anterior and posterior instrumentation with different debridement and grafting procedures for multilevel contiguous thoracic spinal tuberculosis. Orthop Surg 8:454-461, 2016

2. De la Garza RR, Goodwin CR, Abu-Bonsrah N, Bydon A, Witham TF, Wolinsky JP, Sciubba DM: The epidemiology of spinal tuberculosis in the United States: An analysis of 20022011 data. J Neurosurg Spine 26:507-512, 2017

3. Elixhauser A, Steiner C, Harris DR, Coffey RM: Comorbidity measures for use with administrative data. Med Care 36:8-27, 1998

4. Fisahn C, Alonso F, Hasan GA, Tubbs RS, Dettori JR, Schildhauer TA, Rustagi T: Trends in spinal surgery for Pott's Disease (2000-2016): An overview and bibliometric study. Global Spine J 7:821-828, 2017

5. Garg RK, Somvanshi DS: Spinal tuberculosis: A review. J Spinal Cord Med 34:440-454, 2011

6. HCUP CCR. Available at: https://www.hcup-us.ahrq.gov/ toolssoftware/ccs/ccsfactsheet.jsp.

7. He Q, Xu J: Comparison between the antero-posterior and anterior approaches for treating L5 St vertebral tuberculosis. Int Orthop 36:345-351, 2012

8. Hu J, Li D, Kang Y, Pang X, Wu T, Duan C, Cao Y: Active thoracic and spinal tuberculosis in children with kyphotic deformity treated by one-stage posterior instrumentation combine anterior debridement: Preliminary study. Eur J Orthos Surg Traumatol 24 Suppl 1:S221-S229, 2014

9. Kumar K: Spinal tuberculosis, natural history of disease, classification and principles of management with historical perspective. Eur J Orthop Surg Traumatol 26:551-558, 2016

10. Liu J, Wan L, Long Z, Huang S, Dai M, Liu Z: Efficacy and safety of posterior versus combines posterior and anterior approach for the treatment of spinal tuberculosis: A metaanalysis. World Neurosurg 83:1157-1165, 2015

11. Mak KC, Cheung KM: Surgical treatment of acute TB spondylitis: Indications and outcomes. Eur Spine J 22 Suppl 4:641-646, 2013

12. Muheremu A, Niu X, Wu Z, Tian W: Study on anterior and posterior approaches for spinal tuberculosis: A meta-analysis. Eur J Orthop Surg Traumatol 25 Suppl 1:S69-S76, 2015

13. Nagashima H, Yamane K, Nishi T, Teshima R: Recent trends in spinal infections: Retrospective analysis of patients treated during the past 50 years. Int Orthop 34:395-399, 2010

14. Nanzhe Z, Jinhai K, Zhenwang S, Min Q, Tielong L, Jianru X: One-stage posterior approach in the treatment of consecutive multi-segment thoracic tuberculosis with kyphosis. Turk Neurosurg 28(3):439-446, 2018

15. Nationwide Inpatient Sample. Overview of the Nationwide Inpatient Sample (NIS). Available at: http://www.hcup-us. ahrq.gov/nisoverview.jsp. Accessed May 28, 2019 
16. Rajasekaran S: Kyphotic deformity in spinal tuberculosis and its management. Int Orthop 36: 359-365, 2012

17. Yang $\mathrm{P}$, He X, Li H, Zang $Q$, Yang B: Clinical efficacy of posterior versus anterior instrumentation for the treatment of spinal tuberculosis in adults: A meta-analysis. J Orthop Surg Res 9:10, 2014

18. Yang P, Zang Q, Kang J, Li H, He X: Comparison of clinical efficacy and safety among three surgical approaches for spinal tuberculosis: A meta-analysis. Eur Spine J 25:38623874, 2016

19. Yao Y, Zhang H, Liu H, Zhang Z, Tang Y, Zhou Y: Prognostic factors for recovery after anterior debridement/bone grafting and posterior instrumentation for lumbar spinal tuberculosis. World Neurosurg 104:660-667, 2017
20. World Health Organization (WHO) Global Tuberculosis Report 2017. Available at: http://www.who.int/tb/publications/global_ report/gtbr2017_main_text.pdf. Accessed May 20, 2019

21. Wu W, Lyu J, Liu X, Luo F, Hou T, Zhou Q, Li Z, Chen Y, Li LT, Zheng Y, Wang G, Xu J, Zhang Z: Surgical treatment of thoracic spinal tuberculosis-A multi-center retrospective study. J Neurosurg Spine 26:507-512, 2017

22. Zhang X, Ji J, Liu B: Management of spinal tuberculosis: A systematic review and meta-analysis. J Int Med Res 41:13951407, 2013 